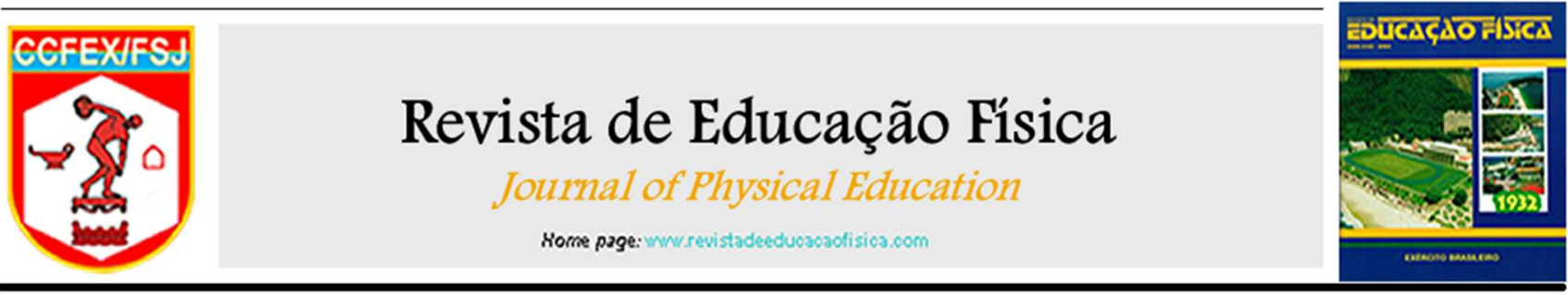

Comentário

Commentary

\title{
Recomendações SOBRASA: "Checklist" individual do atleta para reduzir eventos adversos em águas abertas
}

\section{SOBRASA Statements: Individual Athlete's Checklist to Reduce Adverse Events in Open Water}

\author{
David Szpilman ${ }^{\S 1}$ MD Esp; Danielli Mello² PhD
}

Recebido em: 27 de abril de 2020. Aceito em: 27 de abril de 2020 .

Publicado online em: 21 de maio de 2020.

DOI: $10.37310 /$ ref.v88i4.1336

\section{Resumo}

Introdução: Em 1988, a brasileira Renata Agondi, 25 anos, morreu durante travessia no Canal da Mancha. Em 2010, o americano Fran Crippen, um famoso nadador medalhista em águas abertas, faleceu durante uma prova. Eventos como esses provocaram mudanças na segurança de provas em águas abertas, mas, ainda com resultados insuficientes. Nos últimos anos, observou-se um aumento no número de incidentes e mortes em treinos e competições.

Objetivo: Apresentar o instrumento de avaliação pré-participação em evento esportivo em águas abertas da Sociedade Brasileira de Salvamento (SOBRASA) Trata-se de um auto questionário com respostas simples, no formato "checklist" (lista de checagem), que permite ao atleta avaliar a segurança de sua participação no evento, antes de realizá-lo.

Conclusão: Para desenvolver o questionário, foi formado um grupo focal, constituído por 16 especialistas (profissionais de saúde e do esporte, bombeiros militares e atletas de provas de águas abertas) para se listar e discutir as variáveis de risco envolvidas na realização de uma prova de natação em água abertas, bem como identificar formas para reduzi-los. Mesmo com todas as precauções de segurança que sejam tomadas neste tipo de prova, não há $100 \%$ de segurança. Nesse contexto, cabe tanto ao organizador quanto ao atleta/competidor atentar para os riscos envolvidos na atividade de forma a reduzi-los a fim de prevenir incidentes indesejáveis.

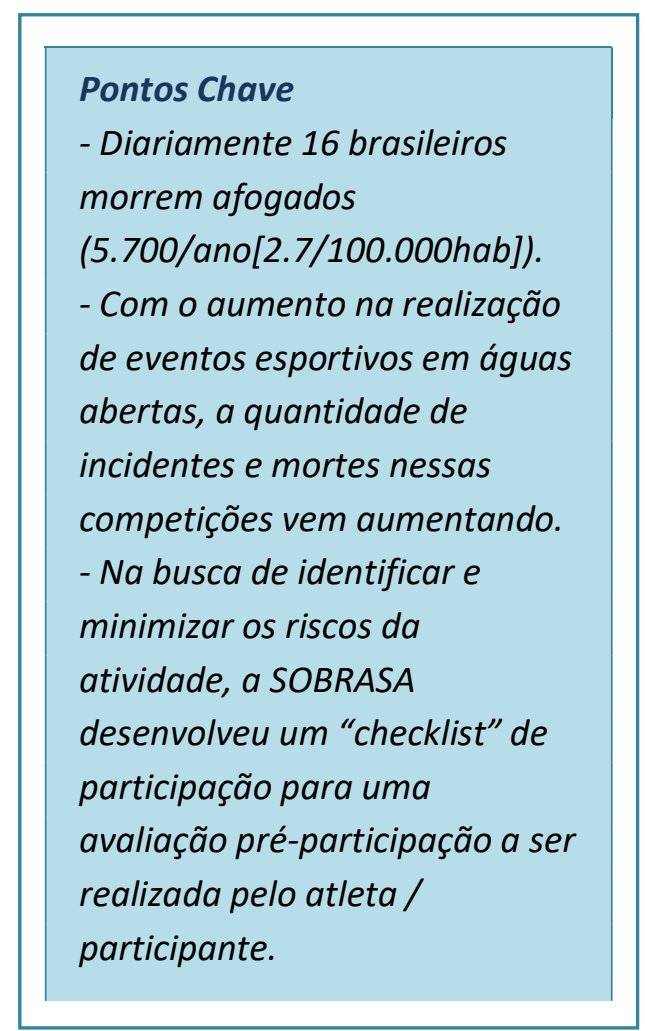

Palavras-chave: afogamento, mortalidade, esportes aquáticos, natação.

\begin{abstract}
Introduction: In 1988, 25-year-old Brazilian Renata Agondi died during swimming across the English Channel. In 2010, the American Fran Crippen, a famous medalist swimmer in open waters, died during a race. Events like these provoked changes in concerns related to safety in open waters events, yet with insufficient results. In recent years, there was an increase in the number of incidents and deaths in training and competitions.
\end{abstract}

$\S$ Autor correspondente: David Szpilman - e-mail: david@szpilman.com

Afiliações: ${ }^{1}$ Sociedade Brasileira de Salvamento Aquático (SOBRASA), Rio de Janeiro - RJ, Brasil; ${ }^{2}$ Escola de Educação Física do Exército (EsEFEx), Rio de Janeiro - RJ, Brasil. 
Objective: To present the pre-participation assessment instrument in a sporting event in open waters of the Brazilian Rescue Society (Sociedade Brasileira de Salvamento Aquático: SOBRASA). It is a selfquestionnaire with simple answers, in the "checklist" format, which allows the athlete to evaluate the security of your participation in the event, before you do it.

Conclusion: In order to develop the questionnaire, a focus group was formed, consisting of 16 specialists (health and sports professionals, military firefighters, and athletes from open water events) to list and discuss the risk variables involved in conducting a test. swimming in open water, as well as identifying ways to reduce them. Even with all the safety precautions that are taken in this type of test, there is no $100 \%$ security. In this context, it is up to both the organizer and the athlete / competitor to pay attention to the risks involved in the activity to reduce them aiming to prevent undesirable incidents.

Keywords: drowning, mortality, water sports, swimming.

\section{Key points}

- Every day 16 Brazilians drown

$(5,700 /$ year $[2.7)$

100,000hab]).

- With the increase in the holding of sporting events in open waters, the number of incidents and deaths in these competitions has been increasing. - In order to identify and minimize the risks of the activity, SOBRASA developed a participation checklist for a preparticipation assessment to be carried out by the athlete / participant.

\section{Recomendações SOBRASA: “Checklist” individual do atleta para reduzir eventos adversos em águas abertas}

Em 1988, a brasileira Renata Agondi, 25 anos, morreu no canal da mancha após $10 \mathrm{~h}$ e 45 minutos durante travessia, quase na chegada. Em 2010, o medalhista, famoso nadador americano, Fran Crippen morreu durante prova em águas abertas, supostamente, por uma suposta arritmia cardíaca provocada pela temperatura alta da água $\left(32^{\circ} \mathrm{C}\right)$. Ambos eram atletas profissionais e faleceram vítimas de afogamento, o que poderia ter sido evitado se houvesse regras de segurança mais rígidas. Após estes eventos, grandes mudanças ocorreram na área de segurança no mundo dos eventos em águas abertas, mas, ainda assim, com o aumento do número de provas e de atletas participantes, a incidência desses eventos adversos tem aumentado.

De acordo com as estatísticas, diariamente, 16 brasileiros morrem afogados (5.700/ano[2.7/100.000hab])(1). No Brasil, o afogamento é a segunda principal causa de óbito na faixa de 1-4 anos; a terceira de 5-14 anos; e a quarta de 15-19 anos. Infelizmente, o Brasil é um dos países com o maior número de óbitos em todo mundo e nossos jovens estão entre as maiores vítimas. $\mathrm{O}$ risco de morte por afogamento, para aqueles dentro ou perto de um espelho d'água, quando comparado ao acidente de trânsito entre aqueles usando um veículo automotor é 200 vezes maior, demonstrando o alto risco de óbito pela exposição ao meio aquático(2).

Todos os esportes estão sujeitos a eventos adversos durante a sua prática, no entanto os esportes aquáticos possuem um dos maiores riscos a vida(3-5). O meio aquático pode se tornar instantaneamente em um ambiente hostil a nossa sobrevida caso ocorra a perda de consciência, por um trauma (mesmo pequeno em região de cabeça) ou um mal súbito. Existem inúmeras possibilidades de eventos adversos, em competições águas abertas, e muito mais frequentes do que temos real conhecimento. Essa falta de conhecimento pode determinar o afogamento em 1 minuto e a morte deste esportista em 5 minutos, caso o processo não seja interrompido imediatamente(2).

$\mathrm{O}$ aumento no número de realizações de eventos esportivos em águas abertas, 
profissionais e amadores, vem crescendo ao longo dos anos, assim como a quantidade de incidentes e mortes nessas competições.

A Sociedade Brasileira de Salvamento (SOBRASA) foi fundada em 1995, por especialistas em salvamento, e tem a missão de unir o Brasil para reduzir a morbimortalidade por afogamento, por meio de programas de prevenção e educação. Seus três pilares principais de ação são: 1) Reunir os profissionais na área de segurança aquática nos estados com a finalidade de trocar informações e debater as melhores práticas de preparação, prevenção, resgate e mitigação de afogamento; 2) Criar e promover campanhas e programas de prevenção em afogamento para todos; e 3) Incentivar e apoiar trabalhos de estudo e pesquisa na área de salvamento aquático visando desenvolver conhecimento científico que contribua para a redução da incidência de afogamentos. Desde a sua fundação, em 1995, foram criados e promovidos mais de 30 programas diferentes de prevenção, abrangendo todos os cenários aquáticos, atividades e idades afetadas pelo afogamento.

A cada ano, muitos brasileiros falecem praticando algum esporte aquático ou durante provas de águas abertas, sendo a ocorrência predominante em homens jovens. Mesmo em um local ou prova com supervisão adequada de guarda-vidas o risco é alto e toda e qualquer forma de reduzi-lo deve ser recomendada. A maioria dos casos de afogamentos pode ser evitada adotando-se medidas de prevenção e redução de riscos e a mais importante delas é a supervisão bem realizada nos ambientes aquáticos. Óbitos por afogamento durante eventos de esportes aquáticos são menos frequentes do que em outras situações; no entanto, quando ocorrem, têm grande repercussão em mídia por, usualmente, tratarem-se de pessoas que sabiam nadar, por vezes, atletas de alto desempenho, demonstrando uma vulnerabilidade inesperada e, supostamente, impossível de ocorrer.

$\begin{array}{crrr}\text { A frase "SABER } & \text { NADAR } & \text { NAOO } & \dot{E} \\ \text { BLINDAGEM } & \text { CONTRA } & & O\end{array}$
AFOGAMENTO"(6), retrata a realidade de que, apesar do aprendizado da natação reduzir a possibilidade imediata de afogamento, por permitir a flutuação e a eventual saída de situações de risco dentro da água, esse processo de aprendizagem demanda muito mais tempo dentro ou ao redor da água, aumentando o risco inerente à exposição . É fundamental o entendimento de que o saber nadar é inteiramente relativo ao local e suas condições de risco (ex.: uma criança que sabe atravessar nadando uma piscina de $25 \mathrm{~m}$, pode se afogar facilmente em uma praia com apenas 0,5 m de ondas), portanto, não só saber nadar é importante, mas, também, compreender os riscos inerentes ao ambiente aquático e conhecer os limites pessoais.

Face ao exposto, é importante que atletas, que pretendem inscrever-se em uma competição esportiva em águas abertas, entender quais os pontos importantes para reduzir a possibilidade de ocorrência de um evento adverso consigo. Alguns desses eventos podem ser minimizados, outros podem ser remanejados e ainda outros podem ser evitados. Cabe a ambos, organizador e ao próprio atleta/competidor atentar para estes riscos de forma a atuar para reduzi-los(7).

O objetivo deste trabalho foi apresentar um auto questionário de pré-participação esportiva para eventos em águas abertas elaborado pela SOBRASA, que utilizando uma linguagem simples e acessível, permita ao atleta de esportes aquáticos, profissional ou amador, avaliar seu nível de preparação em termos de preparação técnica, física, estado de saúde física e médica, psicoemocional, e aspectos de segurança referentes à organização da competição, antes de tomar parte em um evento esportivo em águas abertas.

\section{Métodos}

Diante da ocorrência de óbitos em provas de mar aberto, no ano de 2019, teve início uma discussão dentro do grupo de WhatsApp "Sobrasa Saúde", composto por 256 especialistas na área de segurança pública e saúde (médicos, enfermeiros, profissionais de educação física, e bombeiros militar). A partir dos debates, identificou-se a necessidade de se desenvolver um instrumento de préparticipação em eventos esportivos em águas abertas e o tema foi proposto, na forma de desafio. Posteriormente, foi formado um grupo específico para desenvolvimento do "Checklist de Eventos em águas abertas", que contou com a participação de 16 especialistas: convidados 
do grupo original, interessados em contribuir ao tema e novos integrantes tais como: técnicos, organizadores, atletas profissionais e amadores na área de esportes aquáticos. Durante as discussões no grupo, foram listados todos os itens considerados relevantes de risco e quais seriam as formas de reduzi-los. Todos os itens foram, então, classificados em grandes áreas de interesse na forma de um "checklist" (lista de checagem) que pudesse ser respondido pelo atleta que deseja participar de um evento em águas abertas. Os autores procuraram simplificar ao máximo o questionário com o objetivo de alertar e educar o atleta, que responde questões sobre sua segurança em um evento de águas abertas, seja uma competição, um treino ou apenas natação de lazer. As questões específicas tinham por finalidade orientar o atleta participante a uma tomada de decisão de forma mais consciente antes de se inscrever para participar de uma competição esportiva ou de realizar uma competição em águas abertas. $\mathrm{O}$ instrumento foi proposto utilizando-se uma linguagem informal e acessível por meio de perguntas fechadas, que permitem somente as respostas dicotômicas $\operatorname{sim}(\mathrm{S})$ ou não $(\mathrm{N})$.

\section{0 "Checklist" SOBRASA}

Por consenso, dentro do grupo de especialistas $(\mathrm{n}=16)$, os diversos riscos identificados foram divididos em cinco áreas: preparação técnica e física para participar do evento aquático; características de segurança relativas ao meio ambiente do evento; saúde individual; saúde psicoemocional; e a segurança proporcionada pela organização do evento. Cada área ficou composta por vários itens a serem avaliados pelo próprio atleta, tanto em relação às suas condições de participação quanto às condições oferecidas pelos organizadores do evento. As apresentamse a seguir e o instrumento completo apresentase na Figura 1.

\section{Sua preparação aquática está apropriada ao evento?}

Para avaliar o item "Sua preparação aquática está apropriada ao evento?", foram elaboradas cinco questões, com a finalidade de relevar os cuidados pessoais relativo ao evento aquático proposto. Refere-se à autoavaliação quanto à adequada preparação técnica e física em identificar o nível de treinamento adequado para participar de uma competição em águas abertas; quanto ao estado nutricional nas 24 horas antecedentes à competição / prova; se houve hidratação e ingestão adequada de nutrientes(8); e se houve o cuidado em identificar a adaptação ao traje de neoprene quanto à experiência prévia com o equipamento e se existe a necessidade da utilização, em função da temperatura da água, a fim de evitar casos de hipertermia ou hipotermia.

O local da prova/meio ambiente está seguro para ser executada?

Para avaliar o item "O local da prova/meio ambiente está seguro para ser executada?", foram elaboradas três questões, com a finalidade de identificar o nível de segurança do local da prova/competição quanto as condições meteorológicas (mar / ondas / correntes / ventos); ambientais (temperatura ambiente / água adequados / presença de animais marinhos e substâncias nocivas); e se a prova / local do evento está bem sinalizado e compreendido pelo atleta.

\section{Sua condição médica está adequada à prova?}

Para avaliar o item "Sua condição médica está adequada à prova?", foram elaboradas cinco questões, com a finalidade de identificar a condição médica e nível de risco à saúde de um atleta, amador ou profissional, para realização de um evento esportivo utilizando como ponto de corte a idade de risco para eventos cardiovasculares (35 anos) se há alguma possibilidade de súbito período de inconsciência; se houve uso de drogas (incluindo o álcool); e uma avaliação de saúde autorrelatada ou médica(8).

\section{Seu estado emocional é adequado à prova?}

Para avaliar o item "Seu estado emocional é adequado à prova?", foram elaboradas três questões, com a finalidade de identificar o estado psicoemocional do atleta para participar de uma prova/competição em águas abertas, incluindo condições ambientais desfavoráveis. Questiona-se se o atleta já realizou prova com mesmas características desta, se está seguro/tranquilo para realizar essa prova específica; e se as principais dúvidas que tinha sobre a prova foram devidamente esclarecidas. 


\section{A segurança no evento é adequada à estrutura?}

Para avaliar o item "A segurança no evento é adequada à estrutura?", foram elaboradas seis questões, com a finalidade de identificar a importância de se assegurar estar se inscrevendo / participando em um evento bem organizado no que tange à segurança. Itens tais como: se a organização esclareceu os locais / momentos críticos do percurso, que apresentam risco maior para a ocorrência de afogamento; se a quantidade de participantes de sua prova / largada parece adequada; se existe segurança para socorrer dentro e fora da água; e se o atleta está ciente de como e quando deve agir pedindo ajuda em caso de necessidade.

\section{Conclusão}

Não há ambiente ou prova aquática $100 \%$ segura, mas há como torná-las mais seguras. Dentre mais de 20 variáveis listadas e avaliadas, nenhuma apresentou-se fixa e constante; antes, variam conforme o dia, tanto em relação a condições ambientais quanto pessoais e de segurança, as últimas oferecidas pela organização / elaboração do(a) evento / prova. Portanto, recomenda-se que o atleta preste muita atenção ao responder as perguntas propostas pelo Checklist antes de participar de qualquer evento em águas abertas. Além disso, é de extrema importância que o atleta evite concluir que por já ter nadado aquele percurso antes, as condições de riscos no dia em questão serão as mesmas daquela ocasião e, portanto, os cuidados para avaliar a segurança do evento devem ser os mesmos como se fosse a primeira vez naquele local.

$\mathrm{O}$ grupo elaborou o "Checklist individual para participação em eventos esportivos em águas abertas" no formato visual, que se apresenta na Figura 1, para o qual o atleta deverá procurar solucionar qualquer "NÃO" que tenha respondido, de forma a aumentar sua segurança pessoal naquela prova específica.

Para fins acadêmico-científicos, o "Checklist individual para participação em eventos esportivos em águas abertas" consta na Tabela 1.

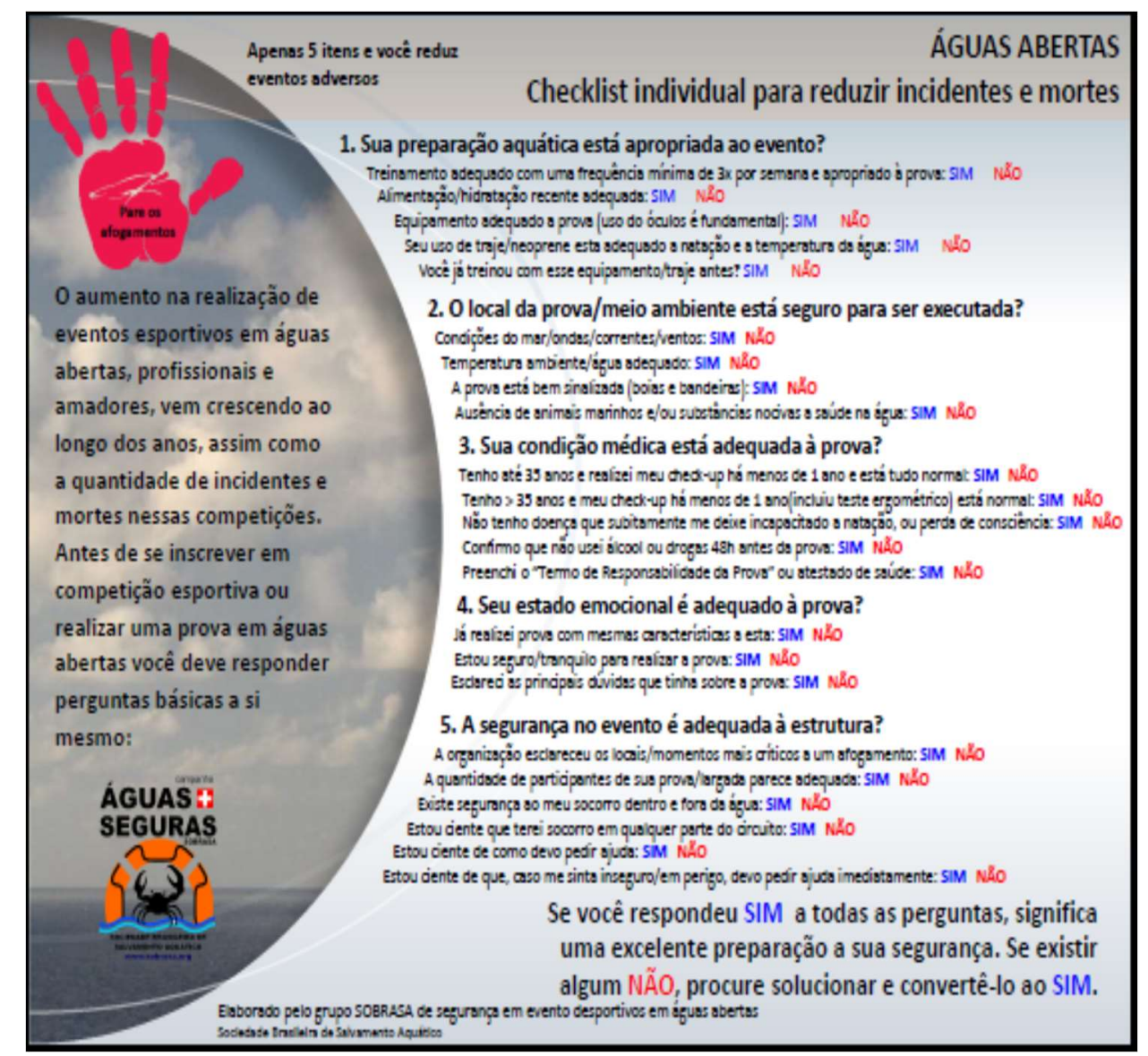

Figura 1 - SOBRASA Checklist individual para participação em eventos esportivos em águas abertas 
Tabela 1 - SOBRASA Águas Abertas - Checklist individual

\section{SOBRASA AGUAS ABERTAS - Checklist individual para reduzir incidentes e mortes}

Sua preparação aquática está apropriada ao evento?

$>$ Treinamento adequado com uma frequência mínima de 3 x por semana e apropriado à prova

> Alimentação/hidratação recente adequada

$>$ Equipamento adequado à prova (uso de óculos é fundamental)

$>\mathrm{O}$ uso de traje/Neoprene está adequado à natação e à temperatura da água

$>$ Você já treinou com esse equipamento antes?

O local da prova/meio ambiente está seguro para ser executada?

$>$ Condições do mar/ondas/correntes/ventos

$>$ Temperatura ambiente/água adequado

$>$ A prova está bem sinalizada (boias e bandeiras)

$>$ Ausência de animais marinhos e/ou substâncias nocivas à saúde na água

Sua condição médica está adequada à prova?

$>$ Tenho até 35 anos e realizei meu check-up há menos de 1 ano e está tudo normal

$>$ Tenho $>35$ anos e meu check-up há menos de 1 ano (incluiu teste ergométrico) está tudo normal

$>$ Não tenho doença que subitamente me deixe incapacitado a natação, ou perda de consciência

$>$ Confirmo que não usei álcool ou droga 48h antes da prova

$>$ Preenchi o "Termo de Responsabilidade da Prova" ou atestado de saúde

Seu estado emocional é adequado à prova?

$>$ Já realizou prova com mesmas características a esta

$>$ Estou seguro/tranquilo para realizar a prova

Esclareci as principais dúvidas que tinha sobre a prova

A segurança no evento é adequada à estrutura?

$>$ A organização esclareceu os locais/momentos mais críticos a um afogamento

$>$ A quantidade de participantes de sua prova/largada parece adequada

$>$ Existe segurança ao meu socorro dentro e fora da água

$>$ Estou ciente que terei socorro em qualquer parte do circuito

$>$ Estou ciente de como devo pedir ajuda

$>$ Estou ciente de que, caso me sinta inseguro/em perigo, devo pedir ajuda imediatamente

Szpilman, D. \& Mello, D. - Rev Ed Fís / J Phys Education. 2020; 88(4): 1034-1040

\section{Agradecimentos}

Agradecimento aos integrantes do Grupo SOBRASA pela contribuição na elaboração do documento: Claudia Coutinho, Claudenir Celistino, Fabrizio Di Masi, Fabrício Vieira, Franz Burini, Glauco Rangel, Lucia Eneida, Magna Costa, Maurício Soares da Silva e Roberto Trindade.

\section{Declaração de conflito de interesses}

Não há nenhum conflito de interesses em relação ao presente estudo.

Declaração de financiamento

Estudo realizado sem nenhum financiamento. 


\section{Referências}

1. SOBRASA, Szpilman D. Afogamento Boletim epidemiológico no Brasil - Ano 2019 (ano base de dados 2017 e outros). [Online] [Accessed: 16th May 2020]. Available from: https://www.sobrasa.org/afogamentoboletim-epidemiologico-no-brasil-ano2019-ano-base-de-dados-2017-e-outros/ [Accessed: 16th May 2020]

2. Szpilman D, Bierens JJLM, Handley AJ, Orlowski JP. Drowning. New England Journal of Medicine. [Online] Massachusetts Medical Society; 2012;366(22): 2102-2110. Available from: doi:10.1056/NEJMra1013317

3. Vago H, Szabo L, Czimbalmos CS, Dohy ZS, Csecs I, Toth A, et al. 4251 Results from the Hungarian Cardiac Magnetic Resonance Registry of Structural Heart Disease and Aborted Sudden Cardiac Death in Athletes. European Heart Journal. [Online] Oxford Academic; 2019;40(Supplement_1). Available from: doi:10.1093/eurheartj/ehz745.0134

[Accessed: 16th May 2020]

4. Wisten A, Borjesson M, Krantz P, Stattin EL. 4100 Exercise related sudden cardiac death in the young in Sweden 2000-2010 a nationwide study showing decreasing incidence in competitive athletes. European Heart Journal. [Online] Oxford Academic; 2019;40(Supplement_1). Available from: doi:10.1093/eurheartj/ehz745.0112

[Accessed: 16th May 2020]

5. Pelliccia A, Adami PE, Caselli S. Olympic athletes and sudden cardiac death risk. European Heart Journal. [Online] Oxford Academic; 2017;38(27): 2092-2094. Available from: doi:10.1093/eurheartj/ehx349

6. Szpilman D, Pinheiro A, Madormo S. Drowning perception risk table. World Conference on Drowning Prevention - ILS. Vancouver; 2017. p. 105.

7. SOBRASA. Recomendação SOBRASA Segurança em evento de águas abertas. [Online] SOBRASA - Sociedade Brasileira de Salvamento Aquático. Available from: http://www.sobrasa.org/recomendacaosobrasa-seguranca-em-evento-de-aguasabertas/ [Accessed: 30th July 2019]

8. American College of Sports Medicine. ACSM's Guidelines for Exercise Testing and Prescription. 10th ed. Philadelphia: LWW; 2017. $480 \mathrm{p}$. 